\title{
Conductometry and Size Characterization of Polypyrrole Nanoparticles Produced by Ball Milling
}

\author{
Abdul Malik Rehan Abbasi, ${ }^{1,2}$ Miroslava Marsalkova, ${ }^{1}$ and Jiri Militky ${ }^{1}$ \\ ${ }^{1}$ Technical University of Liberec, Faculty of textile engineering, Studentska 2, 46117 Liberec, Czech Republic \\ ${ }^{2}$ BUITEMS, Department of textile engineering, 87100 Quetta, Pakistan \\ Correspondence should be addressed to Abdul Malik Rehan Abbasi; rehan_abbaci@hotmail.com
}

Received 14 January 2013; Revised 28 March 2013; Accepted 28 March 2013

Academic Editor: Xiangwu Zhang

Copyright (c) 2013 Abdul Malik Rehan Abbasi et al. This is an open access article distributed under the Creative Commons Attribution License, which permits unrestricted use, distribution, and reproduction in any medium, provided the original work is properly cited.

\begin{abstract}
Polypyrrole (PPy), one of the most extensively investigated conducting polymers, has attracted a great deal of interest because of its good electrical conductivity, environmental stability, and easy synthesis. PPy films were produced by polymerization of pyrrole and tosylate $\left(\mathrm{TsO}^{-}\right)$as dopants in the presence of oxidant $\mathrm{FeCl}_{3}$ and polyethylene glycol $\left(\mathrm{M}_{\mathrm{w}} 8000\right)$ at $-5^{\circ} \mathrm{C}$ for $48 \mathrm{~h}$. High energy milling was carried out at $850 \mathrm{rpm}$ in the dry media with the balls of $10 \mathrm{~mm}$. Particles were then characterized by Scanning Electron Microscope and Dynamic Light Scattering techniques for size distribution, and it was found that the size of PPy particles is a decreasing function of time of milling. Electrical conductivity was measured by preparing a homogenous aqueous dispersion of PPy particles and found as exponential decreasing function of time of milling. The changes occurred in PPy after milling was analyzed by Differential Scanning Calorimetry (DSC) and Fourier transform infrared spectroscopy (FTIR).
\end{abstract}

\section{Introduction}

Polypyrrole (PPy), one of the most extensively investigated conducting polymers, has attracted a great deal of interest because of its environmental stability and easy synthesis along with its good electrical conductivity [1]. Along with its other applications, PPy can be used as conducting filler in insulating polymer matrices in preparation of electrically conducting composites. These composites offer potentials in electronic packaging, EMI shields, display devices, electrodes, and other thermal applications [2-5]. Distribution of PPy particles in polymer matrices, however, becomes an issue. It has been reported that PPy nanoparticles can be effectively dispersed due to large surface area for reactions and highly porous in sol form $[6,7]$ and then they provide good conductivity.

Recent studies also showed that the addition of polymers can influence the conductivity by steric stabilization of the polypyrrole chains [8]. To this end, water-soluble polymers of molecular masses over $20,000 \mathrm{~g} \cdot \mathrm{mol}^{-1}$, such as methyl cellulose, poly(vinyl alcohol-co-acetate), poly(ethylene oxide), poly(vinylpyrrolidone), poly(2-vinylpyridine), and poly(vinylmethylether), were studied. When using these additives, conductivities of a broad range $\left(1 \times 10^{-9}-12 \mathrm{~S} \cdot \mathrm{cm}^{-1}\right)$ have been obtained [8]. Specifically, for poly(ethylene oxide) with a molar mass of $130,000 \mathrm{~g} \cdot \mathrm{mol}^{-1}$, a conductivity of $2 \times$ $10^{-3} \mathrm{~S} \cdot \mathrm{cm}^{-1}$ was found [9]. For this reason PEG 8000 was added in the preparation of PPy.

Currently, various ways to produce PPy nanoparticles have been presented which can provide better electrical conductivity of surface coatings, when applied on any substrate by bottom-up techniques. Their application is based on empirical evidence, and prediction based on their size and electrical conductivity is still very limited. This limits the design of nanocomposite structures with the purpose of their use. The purpose of this study is to produce the nanoparticles of PPy by top-to-bottom technique that is ball milling and characterize their size and electrical conductivity.

\section{Materials and Methodology}

Pyrrole (99\%) was distilled three times before use. $p$-Toluenesulfonic acid (PTSA) as dopant, $\mathrm{FeCl}_{3}$, and polyethylene 


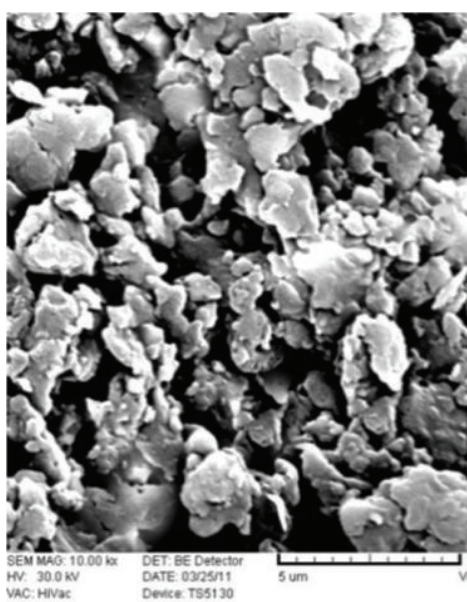

(a)

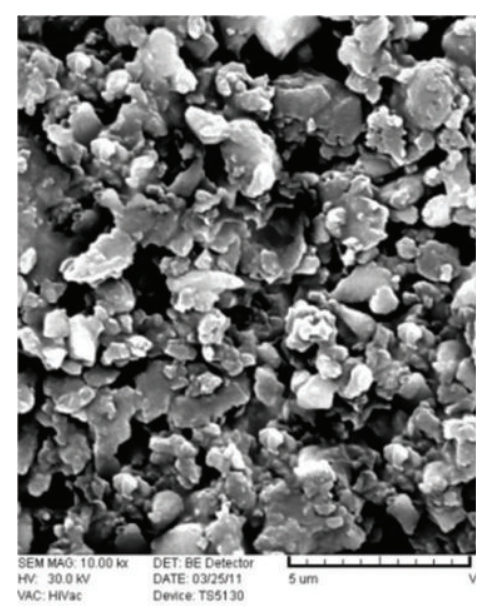

(b)

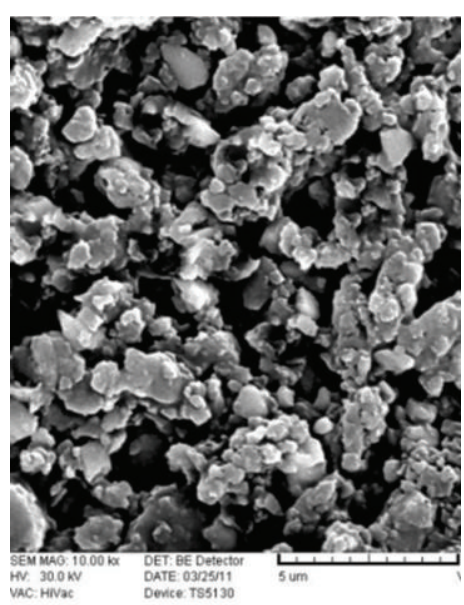

(c)

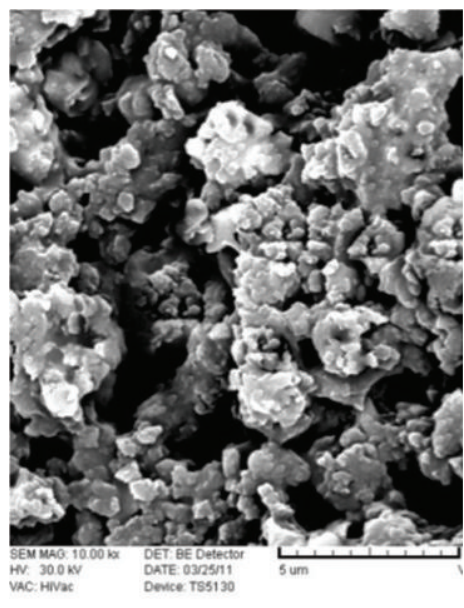

(d)

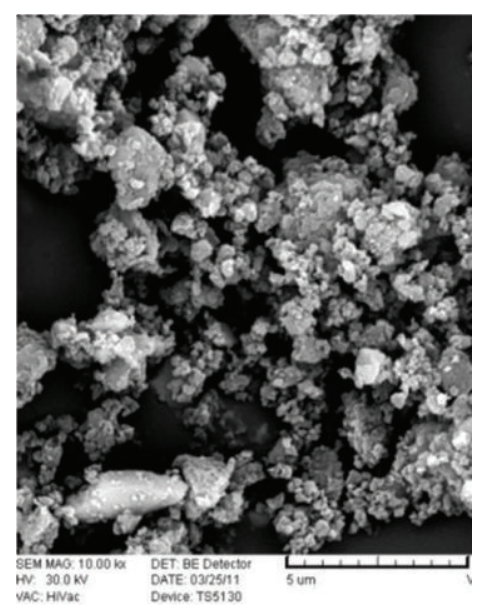

(e)

Figure 1: SEM images of PPy particles taken after (a) $60 \mathrm{~min}$, (b) $120 \mathrm{~min}$, (c) $180 \mathrm{~min}$, (d) $240 \mathrm{~min}$, and (e) $300 \mathrm{~min}$.

glycol (PEG) 8000 were used as received. All of the chemicals were obtained from Sigma-Aldrich.

The molar ratio of monomer (pyrrole), oxidant $\left(\mathrm{FeCl}_{3}\right)$, and dopant (PTSA) was kept at $1: 2: 2 . \mathrm{FeCl}_{3}$ and PTSA were dissolved separately in $50 \mathrm{~mL}$ of distilled water at $0^{\circ} \mathrm{C}$ under constant stirring with gradual addition. After complete dissolution, both solutions are mixed together and temperature was reduced below $0^{\circ} \mathrm{C}$. PEG $8000(20 \mathrm{~g})$ was also dissolved in $30 \mathrm{~mL}$ of distilled water at $25^{\circ} \mathrm{C}$. Solution of PEG was added in it in single step. After $10 \mathrm{~min}$ of severe stirring, distilled pyrrole was added drop by drop with constant high stirring. Temperature was kept around $-5^{\circ} \mathrm{C}$ for $48 \mathrm{~h}$ with constant stirring. Polymer was filtered and washed with distilled water several times and finally 3 times with acetone. The polymer was then dried under vacuum.

Milling was carried out in Planetary Ball Milling machine (FRITSCH PULVERISETTE7 premium line) at $850 \mathrm{rpm}$ at room temperature. Keeping reverse rotations on, milling can be achieved with much more homogenization. Sintered corundum-99.7\% $\mathrm{Al}_{2} \mathrm{O}_{3}$-with steel casing bowls (FRITSCH) and Zirconia balls (FRITSCH) of $10 \mathrm{~mm}$ diameter were selected in this experiment. Bowls were cooled down to room temperature after each 2-minute milling cycle. Ball-to-mass ratio was kept as $6: 1$. Samples were collected for analysis each after 60 minutes of milling till 300 minutes.

Each sample was characterized by SEM by putting the particles on double-sided tape and placed on specimen mount. Specimen mount was then coated with gold particles before recording the images. For precise size distribution measurement $0.1 \mathrm{~g}$ from each milled sample was dispersed in distilled water Bandelin SONOPULS ultrasonic homogenizer for $5 \mathrm{~min}$ and analyzed by DSL (Malvern Instruments' Zetasizer Nano series).

Dispersions of PPy particles in distilled water were prepared under ultrasonic homogenizer for 30 minutes at ambient temperature. Electrical conductivity of the dispersions of $0.5 \%$ to $3 \%$ with gap of $0.5 \%$ was measured by conductometer.

PPy powder after 300 minutes of milling was analyzed by Differential Scanning Calorimetry (DSC) from Perkin Elmer for the glass-transition temperature. Heating rate of $15^{\circ} \mathrm{C} / \mathrm{min}$ and nitrogen flow rate of $20 \mathrm{~mL} / \mathrm{min}$ were set throughout the 


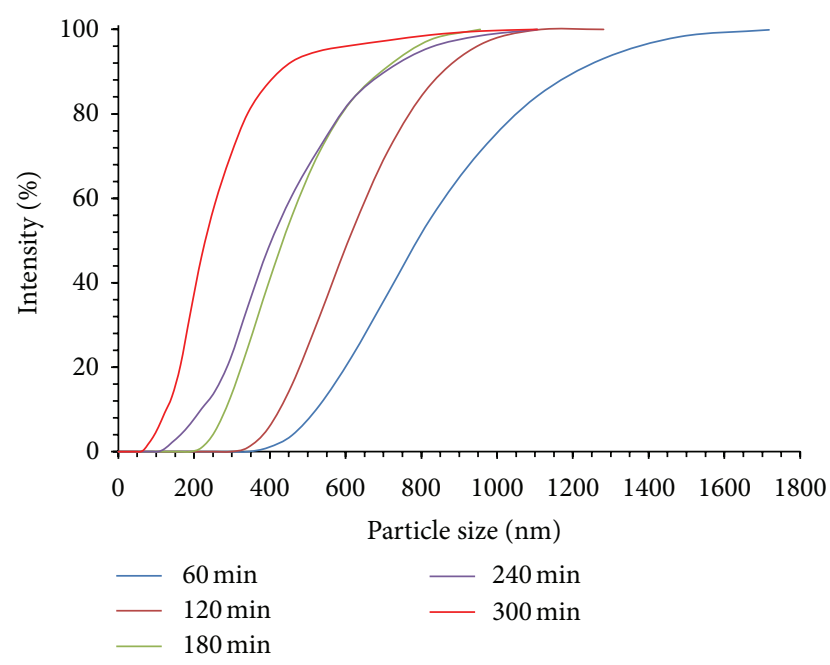

FIGURE 2: Undersize distribution of particles after ball milling.

experiment. The samples are sealed in a standard aluminum pan, and the measurement is done in the temperature range of $25-150^{\circ} \mathrm{C}$. The weight of each sample is about $10 \mathrm{mg}$. The DSC heat flow and temperature values are calibrated with a standard Indium.

The powder after 300 minutes of milling was also analyzed by FTIR spectroscopy in order to observe the changes that occurred due to high energy milling and compared with PPy without milling.

\section{Results}

3.1. Size Characterization. PPy powders were collected after $60,120,180,240$, and 300 min of milling and cooled down to room temperature, that is, $25 \pm 1^{\circ} \mathrm{C}$ at $65 \%$ relative humidity. Small sample from each milled powder was analyzed by SEM as shown in Figure 1.

SEM measurement reveals that the resulting PPy particles size continuously reduces during milling and these have nonspherical morphology with $60-90 \mathrm{~nm}$ in average diameter after 300 minutes of milling.

Decrease in particle size can also be observed from undersize curves of all 5 samples of PPy after milling as shown in Figure 2.

However, the PPy nanoparticles tended to form an aggregation since the average particle size, measured by a DSL nanosizer, was found to be in the range of 78-1100 $\mathrm{nm}$ after 300 minutes of milling. This value was much larger than the particle size observed from SEM images.

3.2. Conductometry. Figure 3 shows an exponential decreasing function of liquid electrical conductivity with increasing milling time of PPy. The relation which has found can be written as

$$
\mathrm{Lc}=K \cdot t^{-0.343} \text {. }
$$

The exponent $(-0.343)$ is found to be valid for all 5 samples with standard deviation of 0.017 . Lc is the liquid electrical

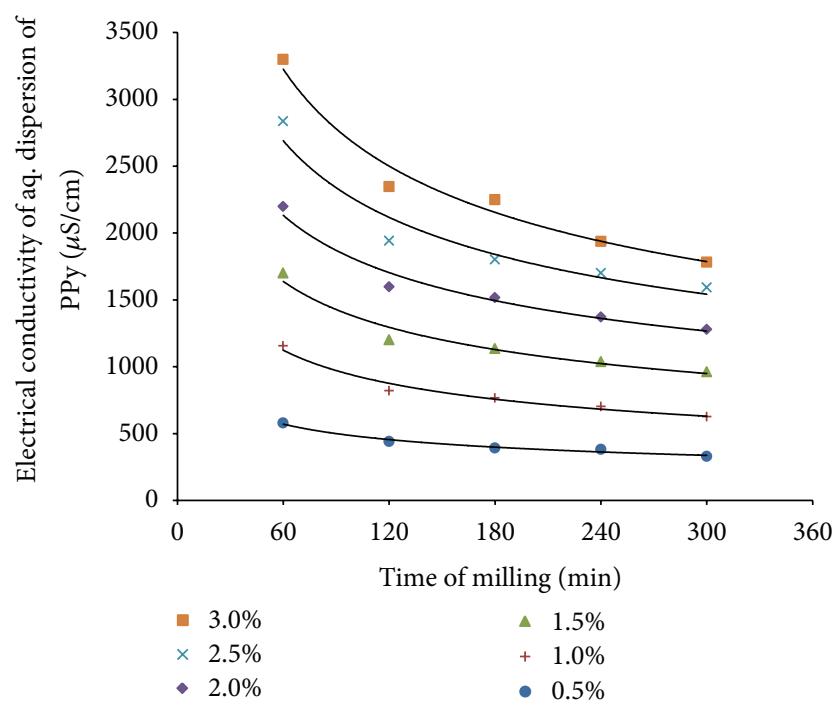

FIGURE 3: Conductometry of milled samples at different concentrations.

conductivity, $t$ is the time of milling, and $K$ is the constant depending upon the concentration of the PPy particles in an aqueous dispersion which is for this experiment found as

$$
K=4662.7 C \text {. }
$$

After 300 minutes of milling, electrical conductivity decreased approximately by 2 times. For example, electrical conductivity of $3 \% \mathrm{w} / \mathrm{v}$ aqueous dispersion of PPy particles after $60 \mathrm{~min}$ of milling was $3298 \mu \mathrm{S} / \mathrm{cm}$ whereas it was measured to be $1783 \mu \mathrm{S} / \mathrm{cm}$ for 300 min milled powder.

3.3. DSC Analysis. Figure 4 shows the DSC of unmilled PPy and particles of PPy after 300 min of milling. Pure PPy shows a broad endothermic crust at $92.3^{\circ} \mathrm{C}$, which might be the glass-transition temperature $\left(T_{g}\right)$ of PPy. The milling of PPy till nanoparticles does not have an effect on the $T_{g}$ of PPy. The second cycle of heating till $150^{\circ} \mathrm{C}$ clearly shows that there were major changes happening during milling.

3.4. FTIR Analysis. The PPy powders prepared after $4 \mathrm{~h}$ of milling and films without milling were analyzed by FTIR as shown in Figure 5. FTIR spectra showed the main characteristic peaks at $1546 \mathrm{~cm}^{-1}$ and $1421 \mathrm{~cm}^{-1}$ corresponding to the fundamental vibrations of polypyrrole ring. The band at $1311 \mathrm{~cm}^{-1}$ corresponds to $\mathrm{C}-\mathrm{H}$ deformation. Other low intensity peaks are observed at around $2892 \mathrm{~cm}^{-1}-2846 \mathrm{~cm}^{-1}$ which can be attributed to aromatic $\mathrm{C}-\mathrm{H}$ stretching vibrations and shows the presence of $\mathrm{TsO}^{-}$doping agent in the structure. However this peak almost vanished in the case of milled PPy powder which confirms the loss in doping agent during milling process. The peaks at 1546 and $781 \mathrm{~cm}^{-1}$ represent $\mathrm{C}=\mathrm{N}$ and $\mathrm{C}-\mathrm{N}$ bonds, whereas the bond of $\mathrm{C}-\mathrm{H}$ in plane deformation vibration is situated at $965 \mathrm{~cm}^{-1}$ in our spectrum. The overlapped peaks at $1054 \mathrm{~cm}^{-1}$ and $1033 \mathrm{~cm}^{-1}$ can be credited to the sulphonate group present in $\mathrm{TsO}^{-}$ 


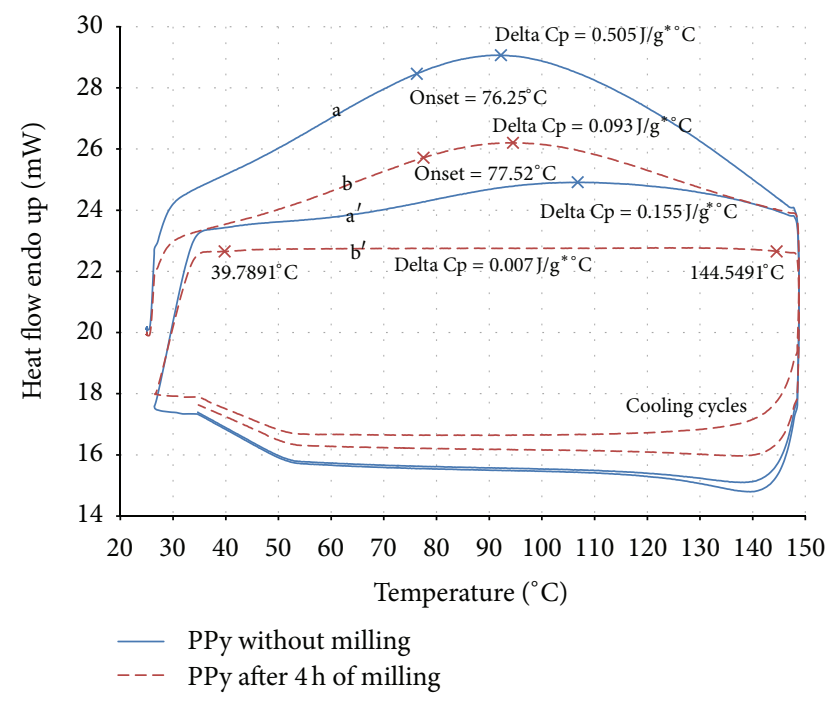

FIgURE 4: DSC plots of unmilled PPy and PPy powders after $4 \mathrm{~h}$ of milling, (a) and $\left(a^{\prime}\right)$ are first and second heating cycles of unmilled PPy, (b) and $\left(b^{\prime}\right)$ are first and second cycles of heating of milled PPy particles, respectively.

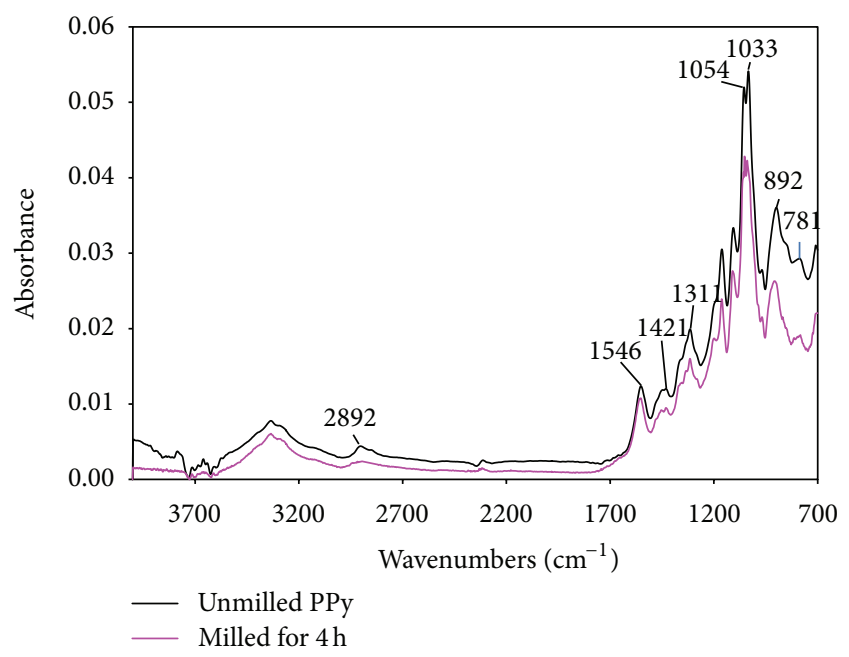

Figure 5: FTIR analyses of unmilled PPy and PPy particles after $4 \mathrm{~h}$ of milling.

which is observed to be lost absorbance considering milled PPy.

\section{Conclusion}

The use of PEG during oxidative polymerization gave much more electrical conductive PPy. After planetary ball milling of PPy polymer pallets up till 300 minutes, nonspherical shaped nanoparticles can be obtained. The disadvantage of achieving the size of the particle in nanometer range is the exponential loss of electrical conductivity of PPy particles. This might happen due to loss of dopant during rise in temperature of milled material in the process of high energy milling as observed in FTIR analysis. Differential Scanning Calorimetry was also found to be a useful tool to explore this fact, but from DSC analysis it can also be concluded that ball milling does not change the $T_{g}$ of PPy.

Planetary ball milling method is still the simplest, easiest, and cheapest method to produce nanoparticles, but results show that size distribution is very broad as well as there is a high chance of agglomeration when bringing particles into ambient conditions. This happens due to dry conditions in the milling process of PPy. Planetary ball mills are useful for small amounts of nanoparticles. These are energy intensive, but the rate of acquisition of the product is much higher compared to other milling machines. The loss of electrical conductivity after milling can be gotten back by redoping and information provided in (1) could be very helpful for it. Nonconductive PPy nanoparticles can be used as thermal transducer of NIR radiation in hot-melt adhesives [10].

\section{Acknowledgment}

This work was financed by Student Grant Scheme (SGS) at the Technical University of Liberec, Czech Republic.

\section{References}

[1] G. Tourillon and F. Garnier, "New electrochemically generated organic conducting polymers," Journal of Electroanalytical Chemistry, vol. 135, no. 1, pp. 173-178, 1982.

[2] C. W. Lin, B. J. Hwang, and C. R. Lee, "Sensing behaviors of the electrochemically co-deposited polypyrrole-poly(vinyl alcohol) thin film exposed to ammonia gas," Materials Chemistry and Physics, vol. 58, no. 2, pp. 114-120, 1999.

[3] H. P. De Oliveira, C. A. S. Andrade, and C. P. De Melo, "Optical and dielectric properties of polypyrrole nanoparticles in a polyvinylalcohol matrix," Synthetic Metals, vol. 155, no. 3, pp. 631-634, 2005.

[4] Y. Wang and X. Jing, "Intrinsically conducting polymers for electromagnetic interference shielding," Polymers for Advanced Technologies, vol. 16, no. 4, pp. 344-351, 2005.

[5] A. M. R. Abbasi, M. Mushtaq Mangat, V. K. Baheti, and J. Militky, "Electrical and thermal properties of polypyrrole coated cotton fabric," Vlakna a Textil, vol. 19, pp. 48-52, 2012.

[6] J. Jang, "Conducting polymer nanomaterias and their applications," Emissive Materials Nanomaterials, vol. 199, pp. 189-260, 2006.

[7] K. Suri, S. Annapoorni, R. P. Tandon, C. Rath, and V. K. Aggrawal, "Thermal transition behaviour of iron oxidepolypyrrole nanocomposites," Current Applied Physics, vol. 3, no. 2-3, pp. 209-213, 2003.

[8] B. M. Mandal, P. Banerjee, and S. N. Bhattacharyya, "Polypyrrole (processable dispersions)," in Polymeric Materials Encyclopedia, J. C. Salamone, Ed., pp. 6670-6678, CRC Press, Boca Raton, Fla, USA, 1996.

[9] S. P. Armes, M. Aldissi, G. C. Idzorek et al., "Particle size distributions of polypyrrole colloids," Journal of Colloid And Interface Science, vol. 141, no. 1, pp. 119-126, 1991.

[10] F. Li, M. A. Winnik, A. Matvienko, and A. Mandelis, "Polypyrrole nanoparticles as a thermal transducer of NIR radiation in hot-melt adhesives," Journal of Materials Chemistry, vol. 17, no. 40, pp. 4309-4315, 2007. 



Submit your manuscripts at http://www.hindawi.com
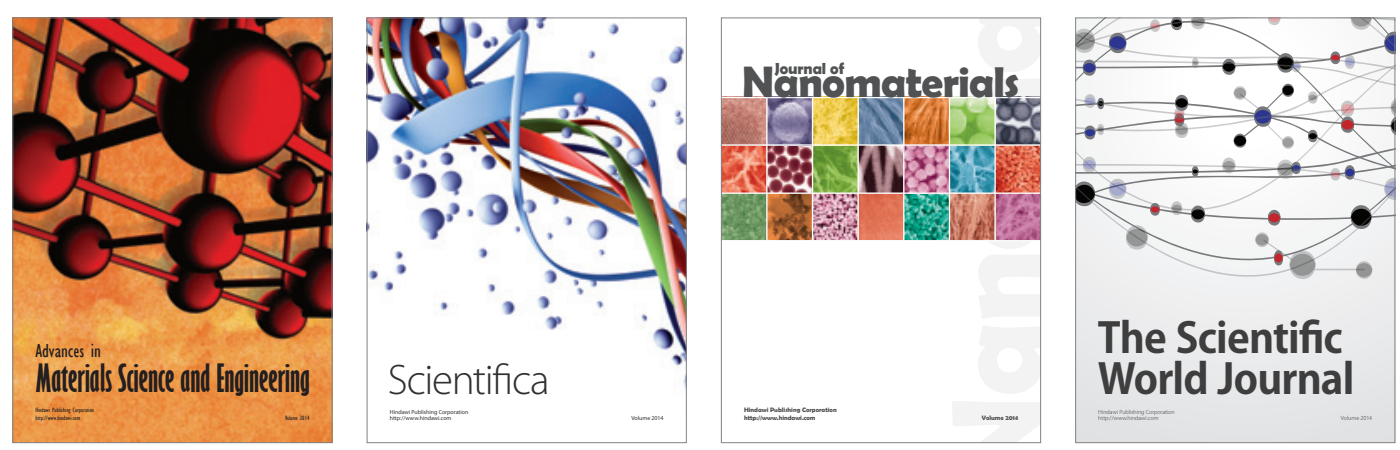

\section{The Scientific World Journal}
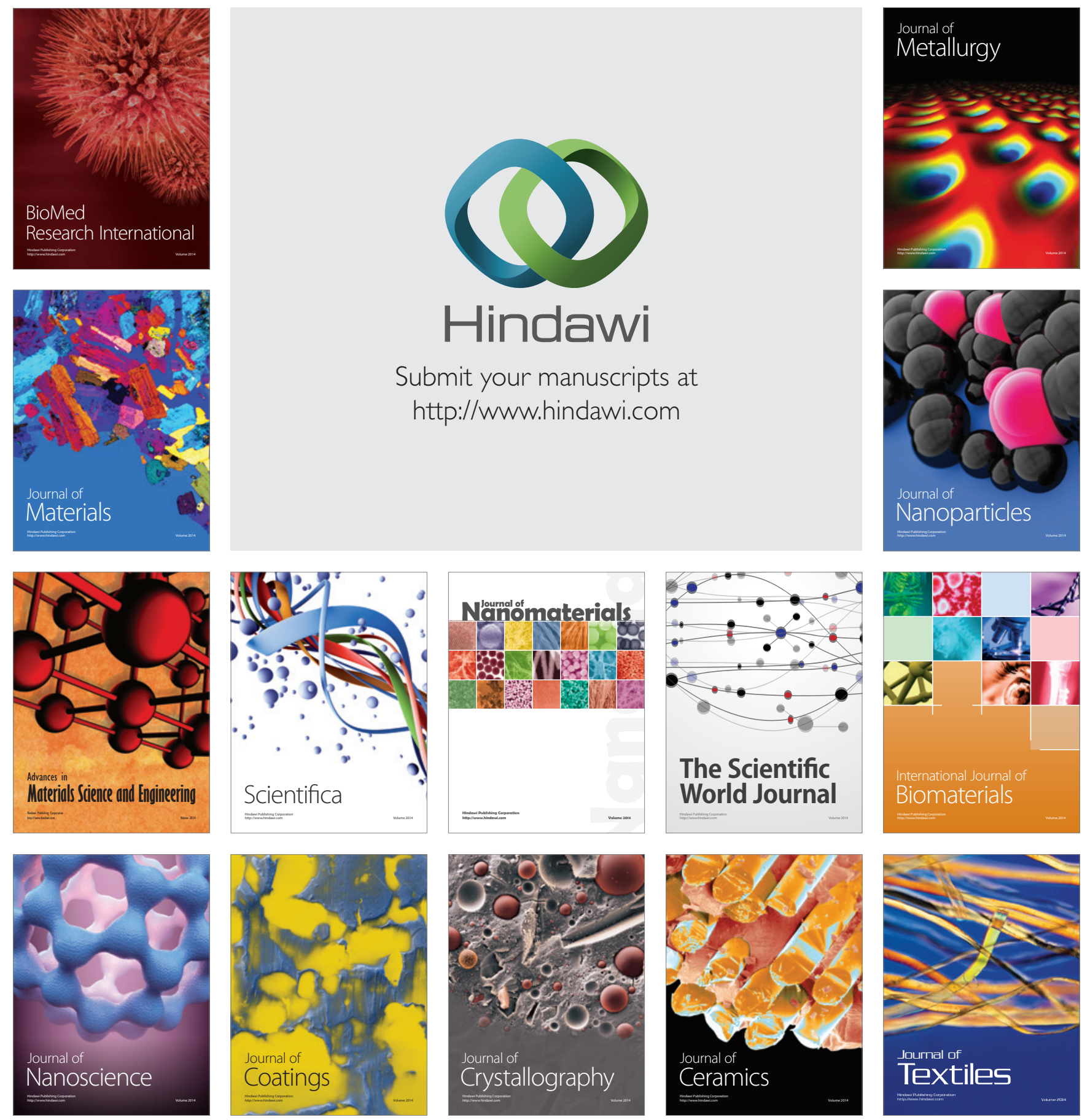